\title{
A SYSTEM OF NONLINEAR VOLTERRA EQUATIONS WITH BLOW-UP SOLUTIONS
}

\author{
C.M. KIRK, W.E. OLMSTEAD AND C.A. ROBERTS \\ Communicated by Hermann Brunner
}

\begin{abstract}
A pair of coupled nonlinear Volterra equations are examined for solutions that can have either global or blow-up behavior. The results represent an extension of the work in [8], where the analysis was restricted to cases in which the integral equations had identical kernels. Sufficient conditions are provided for global solutions as well as for blowup solutions. In the case of blow-up solutions, bounds on the blow-up time are derived. To demonstrate the applicability of the results, two examples are analyzed.
\end{abstract}

1. Introduction. We consider the possible blow-up behavior of the following system of nonlinearly coupled Volterra equations:

$$
\begin{aligned}
& u_{1}(t)=\int_{0}^{t} k_{1}(t-s) F_{1}\left[u_{2}(s)+h_{2}(s)\right] d s, \\
& u_{2}(t)=\int_{0}^{t} k_{2}(t-s) F_{2}\left[u_{1}(s)+h_{1}(s)\right] d s, \quad t \geq 0 .
\end{aligned}
$$

In (1), it is assumed that the kernels $k_{j}(t), j=1,2$, are continuous and differentiable for $t>0$ and have the properties

$$
k_{j}(t) \geq 0, \quad k_{j}^{\prime}(t) \leq 0, \quad 0 \leq t<\infty, j=1,2 .
$$

The given functions $h_{j}(t), j=1,2$, are continuously differentiable for $t \geq 0$ and have the properties

$$
0<a \leq h_{j}(t) \leq b<\infty, \quad h_{j}^{\prime}(t) \geq 0, t \geq 0, j=1,2 .
$$

The nonlinearities $F_{j}(v), j=1,2$, are twice continuously differentiable and have the properties

$$
F_{j}(v)>0, \quad F_{j}^{\prime}(v)>0, \quad F_{j}^{\prime \prime}(v) \geq 0 \text { for } v>0, j=1,2 .
$$

\footnotetext{
Received by the editors on November 4, 2011, and in revised form on March 16, 2012. 
Our goal is to explore the conditions under which the solution $u_{j}(t)$, $j=1,2$, of (1) does or does not exhibit blow-up behavior. In those circumstances where a blow-up does occur, bounds on the blow-up time will be provided.

The general results derived here represent an extension of those presented by Olmstead, Roberts and Deng in $[\mathbf{8}]$. The results presented in [8] apply to system (1) only for the special case in which $k_{1}(t)=$ $k_{2}(t), t \geq 0$.

The relationship of (1) with certain reaction-diffusion problems governed by coupled parabolic partial differential equations is developed in [8]. It is shown how the kernels in (1) are derived from Green's functions associated with the parabolic operators that model the diffusive behavior of the reactive materials. For situations in which the diffusive media have dissimilar properties, the corresponding Green's functions presented in [8] will yield non-equal kernels as allowed in (1).

To illustrate the general results derived here, two examples will be presented. One example that always exhibits a blow-up arises from the work of Kirane and Malik in [3], where a system of fractional differential equations with power-law nonlinearities is analyzed. A second example demonstrates the possibility of either a global or a blow-up solution depending upon the parameters of the problem. A special case of this second example is related to an integro-differential equation being considered by Kirane and Rihani [4].

2. Global and blow-up solutions. It is convenient to express system (1) in vector operator form as

$$
u(t)=T u(t),
$$

where $u=\left[u_{1}, u_{2}\right], k=\left[k_{1}, k_{2}\right], F=\left[F_{1}, F_{2}\right], T=\left[T_{1}, T_{2}\right]$ and

$$
\begin{gathered}
T_{j} u(t)=\int_{0}^{t} k_{j}(t-s) F_{j}\left[u_{3-j}(s)+h_{3-j}(s)\right] d s, \\
\quad \text { for } 0 \leq t<\hat{t}, \quad j=1,2 .
\end{gathered}
$$

In order to examine (3) for both the existence and nonexistence of a global solution, we employ a methodology similar to that of $[8]$. We consider the following space of pairs of continuous functions:

$$
X=\left\{u \mid u_{j} \text { continuous; } 0 \leq u_{j}(t) \leq M<\infty, 0 \leq t<\hat{t}, j=1,2\right\},
$$


with the norm

$$
\|u\|=\sum_{n=1}^{2} \sup _{0 \leq t<\hat{t}}\left|u_{n}(t)\right| .
$$

To explore existence, we will apply the contraction mapping theorem in order to establish a unique fixed point $u \in X$. It is clear that $T_{j} u(t)$ is continuous and that

$$
0 \leq T_{j} u(t) \leq F_{j}(M+b) I_{j}(t), \quad 0 \leq t \leq \hat{t}, j=1,2
$$

where

$$
I_{j}(t)=\int_{0}^{t} k_{j}(s) d s, \quad j=1,2 .
$$

In order for $T$ to map $X$ into itself, it is sufficient that

$$
F_{j}(M+b) I_{j}(\hat{t}) \leq M, \quad j=1,2 .
$$

To satisfy this for $j=1,2$, it is sufficient to require

$$
\bar{F}(M+b) \bar{I}(\hat{t}) \leq M
$$

where

$$
\begin{aligned}
\bar{F}(M+b) & =\max _{j=1,2} F_{j}(M+b), \\
\bar{I}(t) & =\int_{0}^{t} \bar{k}(s) d s, \quad \text { and } \\
\bar{k}(t) & =\max _{j=1,2} k_{j}(t) .
\end{aligned}
$$

In order that $T$ has the contraction property, it will be shown to be sufficient that

$$
\bar{F}^{\prime}(M+b) \bar{I}(t)<1,
$$

where $\bar{F}^{\prime}(M+b)=\max _{j=1,2} F_{j}^{\prime}(M+b)$.

To satisfy both (4) and (6), it is appropriate to define

$$
\Lambda=\frac{M^{*}}{\bar{F}\left(M^{*}+b\right)}, \quad M^{*} \bar{F}^{\prime}\left(M^{*}+b\right)=\bar{F}\left(M^{*}+b\right) .
$$


Satisfaction of (4) and (6) holds for $0 \leq t<t^{*}$, where

$$
\bar{I}\left(t^{*}\right)=\Lambda .
$$

The results on existence of a solution to (1) are given by the following theorem.

Theorem 2.1. System (1) has a unique solution $u(t) \in X$ for $0 \leq t<t^{*}$, where $t^{*}$ is given by (8). If $\bar{I}(\infty)<\Lambda$, then (1) has a unique global solution.

Proof. $T$ maps $X$ into itself whenever (1) is satisfied. To examine the contraction property of $T$, it follows from (3) that

$$
\begin{gathered}
\left|T_{j} u(t)-T_{j} v(t)\right| \leq F_{j}^{\prime}(M+b) \int_{0}^{t} k_{j}(t-s)\left|u_{3-j}(s)-v_{3-j}(s)\right| d s, \\
j=1,2 .
\end{gathered}
$$

Then it follows that

$$
\|T u-T v\| \leq \bar{F}^{\prime}(M+b) \bar{I}(t)\|u-v\| .
$$

Thus, $T$ is a contraction for $0 \leq t<\hat{t}$ provided that (6) is satisfied. The largest interval $\left[0, t^{*}\right)$ for which (4) and (6) are both satisfied is provided by (7) and (8). This assures the existence of a unique solution $u(t) \in X$ for $0 \leq t<t^{*}$.

If $\bar{I}(\infty)<\Lambda$, then (4) and (6) are both satisfied for $0 \leq t<\infty$. Thus, (1) has a global solution.

To demonstrate the monotone growth of $u_{j}(t), j=1,2$, whenever $u(t)$ exists, consider the following theorem.

Theorem 2.2. Whenever (1) has a unique solution $u(t) \in X$, then it is differentiable and $u_{j}^{\prime}(t)>0, j=1,2,0<t<\hat{t}$. 
Proof. The formal derivative of (1) can be expressed in component form as

$$
\begin{aligned}
u_{j}^{\prime}(t)= & k_{j}(t) F_{j}\left[h_{3-j}(0)\right] \\
& +\int_{0}^{t} k_{j}(t-s) F_{j}^{\prime}\left[u_{3-j}(s)+h_{3-j}(s)\right] \\
& \times\left[u_{3-j}^{\prime}(s)+h_{3-j}^{\prime}(s)\right] d s \\
0< & t<\hat{t}, \quad t \geq 0, j=1,2 .
\end{aligned}
$$

This can be viewed as a linear Volterra system for $u_{j}^{\prime}(t), j=1,2$, with the kernels

$$
K_{j}(t, s)=k_{j}(t-s) F_{j}^{\prime}\left[u_{3-j}(s)+h_{3-j}(s)\right], \quad j=1,2 .
$$

The properties of $k_{j}(t), h_{j}(t)$ and $F_{j}^{\prime}(v)$ together with the existence of continuous $u_{3-j}(t), 0 \leq t<\hat{t}$, are sufficient to ensure that $K_{j}(t, s)$ is continuous in both $t$ and $s, 0 \leq s<t<\hat{t}$, with the possibility of an integrable singularity as $s \rightarrow t$. This is sufficient (see [2]) to provide that there is a continuous solution $u_{j}^{\prime}(t), j=1,2$, of (11) for $0<t<\hat{t}$.

To see that $u_{j}^{\prime}(t)>0, j=1,2,0<t<\hat{t}$, first note that $u_{j}(0)=0$ and $u_{j}(t)>0,0<t<\hat{t}$. This implies that $u_{j}^{\prime}(t)>0, j=1,2$, at least on some common small interval $0<t<\bar{t}$. Assume, for example, that $u_{1}^{\prime}(\bar{t})=0, u_{2}^{\prime}(\bar{t}) \geq 0$. Then, from (11) with $j=1$,

$$
\begin{aligned}
0= & k_{1}(\bar{t}) F_{1}\left[h_{2}(0)\right] \\
& +\int_{0}^{\bar{t}} k_{1}(\bar{t}-s) F_{1}^{\prime}\left[u_{2}(s)+h_{2}(s)\right]\left[u_{2}^{\prime}(s)+h_{2}^{\prime}(s)\right] d s .
\end{aligned}
$$

Clearly this is a contradiction since the right side of (12) must be positive for $\bar{t}<\infty$. Thus, $u_{1}^{\prime}(\bar{t}) \neq 0$, and analogously it follows that $u_{2}^{\prime}(\bar{t}) \neq 0$. Hence, no such $\bar{t}$ exists, and consequently, $u^{\prime}=\left[u_{1}^{\prime}, u_{2}^{\prime}\right]$ is such that

$$
u_{j}^{\prime}(t)>0, \quad 0<t<\hat{t}, \quad j=1,2
$$

To investigate the possibility of a blow-up solution to (1), a lower bound on $u(t)$ will be derived. It will be shown that the lower bounding function cannot exist for $t \geq t^{* *}$ if there exists a $t^{* *}<\infty$ such that

$$
\widetilde{I}\left(t^{* *}\right)=\varkappa=\min _{j=1,2} \kappa_{j}
$$


where

$$
\widetilde{I}(t)=\int_{0}^{t} \widetilde{k}(s) d s, \quad \widetilde{k}(t)=\min _{j=1,2} k_{j}(t)
$$

and

$$
\kappa_{j}=\int_{a}^{\infty} \frac{d z}{F_{j}\left[G_{j}^{-1}\left[G_{3-j}(z)+G_{j}(a)-G_{3-j}(a)\right]\right]}, \quad j=1,2 .
$$

The functions $G_{j}(z)$ are the antiderivatives of $F_{j}(z)$, i.e.,

$$
F_{j}(z)=\frac{d G_{j}(z)}{d z}, \quad j=1,2 .
$$

The results on a blow-up solution of (1) are given by the following theorem.

Theorem 2.3. If there exists a $t^{* *}<\infty$ such that (14) is satisfied, then the solution of (1) ultimately experiences a blow-up for some $t_{c}<\infty, t^{*} \leq t_{c} \leq t^{* *}$. The upper and lower bounds on $t_{c}$ are given by (8) and (14), respectively.

Proof. Assume that $u=T u$ has a continuous solution for $0 \leq t \leq \hat{t}$. Since $k_{1}(t)$ and $k_{2}(t)$ are nonincreasing functions, then

$$
u_{j}(t) \geq J_{j}(t) \equiv \int_{0}^{t} k_{j}(\hat{t}-s) F_{j}\left[u_{3-j}(s)+h_{3-j}(s)\right] d s, \quad j=1,2 .
$$

Then

$$
\begin{aligned}
J_{j}^{\prime}(t) & =k_{j}(\hat{t}-t) F_{j}\left[u_{3-j}(t)+h_{3-j}(t)\right] \\
& \geq \widetilde{k}(\hat{t}-t) F_{j}\left[J_{3-j}(t)+a\right], \quad J_{j}(0)=0 .
\end{aligned}
$$

Note that $\widetilde{k}(t)$ is a nonincreasing function since $k_{j}^{\prime}(t) \leq 0$ for $j=1,2$. Also note that a blow-up of either $J_{j}(t)$ would imply a blow-up of the corresponding $u_{j}(t)$. To investigate the possibility of a blow-up in $J_{j}(t)$, consider the following comparison problem:

$$
\begin{gathered}
V_{j}^{\prime}(t)=\widetilde{k}(\hat{t}-t) F_{j}\left[V_{3-j}(t)\right], \quad V_{j}(0)=a-\delta>0, \\
\delta>0, \quad 0 \leq t \leq \hat{t}, j=1,2 .
\end{gathered}
$$


Define

$$
U_{j}(t)=J_{j}(t)+a-V_{j}(t)
$$

Then

$$
\begin{aligned}
& U_{j}^{\prime}(t)=J_{j}^{\prime}(t)-V_{j}^{\prime}(t) \\
& \geq \widetilde{k}(\hat{t}-t)\left[F_{j}\left[J_{3-j}(t)+a\right]-F_{j}\left[V_{3-j}(t)\right]\right] \\
& \geq \widetilde{k}(\hat{t}-t) F_{j}^{\prime}\left[\widetilde{\Theta}_{3-j}(t)\right] U_{3-j}(t), \\
& \quad U_{j}(0)=\delta, \quad j=1,2 .
\end{aligned}
$$

Here $\widetilde{\Theta}_{j}$ lies between $J_{j}+a$ and $V_{j}$ and hence is positive for $j=1,2$. Integration gives

$$
\begin{gathered}
U_{j}(t) \geq \delta+\int_{0}^{t} \widetilde{k}(\hat{t}-s) F_{j}^{\prime}\left[\widetilde{\Theta}_{3-j}(s)\right] U_{3-j}(s) d s, \\
0 \leq t \leq \hat{t}, \quad j=1,2
\end{gathered}
$$

and that $U_{j}(t)>0$ for $0 \leq t \leq \hat{t}$ for all $\delta>0$. Hence, $U_{j}(t) \geq 0$ for $\delta=0,0 \leq t \leq \hat{t}$. Thus, for $\delta=0$,

$$
u_{j}(t) \geq J_{j}(t) \geq V_{j}(t)-a, \quad 0 \leq t \leq \hat{t}, \quad j=1,2 .
$$

Then the system for $V_{j}(t)$ given by (18) can be expressed as

$$
\begin{aligned}
\frac{d}{d t}\left[G_{j}\left(V_{3-j}(t)\right)\right] & =\frac{d}{d V_{3-j}}\left[G_{j}\left(V_{3-j}\right)\right] \frac{d V_{3-j}}{d t} \\
& =F_{j}\left(V_{3-j}\right) \frac{d V_{3-j}}{d t} \\
& =\widetilde{k}(\hat{t}-t) F_{j}\left(V_{3-j}(t)\right) F_{3-j}\left(V_{j}(t)\right), \quad j=1,2 .
\end{aligned}
$$

Since the last expression in (20) is invariant for $j=1,2$, then

$$
G_{2}\left(V_{1}(t)\right)-G_{1}\left(V_{2}(t)\right)=G_{2}(a)-G_{1}(a), \quad 0 \leq t \leq \hat{t} .
$$

Since $F_{j}(V)>0$ for $V>0$, then $G_{j}(V)$ is strictly increasing and hence invertible. Then it follows that

$$
V_{j}(t)=G_{3-j}^{-1}\left[G_{j}\left(V_{3-j}(t)\right)+G_{3-j}(a)-G_{j}(a)\right], \quad j=1,2 .
$$


Thus, from (18) with $\delta=0$, we have

$$
\frac{d V_{j}}{d t}=\widetilde{k}(\hat{t}-t) F_{j}\left[G_{j}^{-1}\left[G_{3-j}\left(V_{j}(t)\right)+G_{j}(a)-G_{3-j}(a)\right]\right]
$$

with $V_{j}(0)=a, 0 \leq t \leq \hat{t}, j=1,2$. Then

$$
\int_{a}^{V_{j}(\hat{t})} \frac{d z}{F_{j}\left[G_{j}^{-1}\left[G_{3-j}(z)+G_{j}(a)-G_{3-j}(a)\right]\right]}=\widetilde{I}(\hat{t}), \quad j=1,2,
$$

where $\widetilde{I}(t)$ is given by (15). If there exists a $t^{* *}<\infty$ such that (14) holds, then (22) implies that $V_{j}(t) \rightarrow \infty$ as $t \rightarrow t^{* *}$. In view of (19), this implies that $u_{j}(t) \rightarrow \infty$ as $t \rightarrow t_{c} \leq t^{* *}$.

The lower bound on $t_{c}$ follows from Theorem 2.1, which provides that $u(t)$ exists for $0 \leq t<t^{*}$.

The results presented here for blow-up solutions of the Volterra system (1) can be compared to very recent work in [1] for a single Volterra equation. Similar to Theorem 2.3, the results of [1] provide sufficient conditions for blow-up that include properties of the kernel to ensure that $I(t)$ grows sufficiently fast while a measure of the strength of the nonlinearity, analogous to $\kappa$, remains bounded. In $[\mathbf{1}]$, the main theorems are applied to various known blow-up problems to demonstrate the versatility of the results.

Recent work in [7] examines the blow-up time for solutions to some nonlinear Volterra equations. Here again the growth of $I(t)$ and its inverse, along with the strength of the nonlinearity, are used to develop bounds on the blow-up time. Other recent results on blow-up behavior for nonlinear Volterra equations are reviewed in $[\mathbf{9}]$.

3. Application to a system with power-law nonlinearities. In this section, we consider an example of system (1) studied in [3]. As part of the analysis in $[\mathbf{3}]$, the authors consider the following system of nonlinear Volterra equations:

$$
\begin{array}{ll}
u(\tau)=u_{0}+\frac{\mu}{\Gamma(\alpha)} \int_{0}^{\tau} \frac{[v(\sigma)]^{q}}{(\tau-\sigma)^{1-\alpha}} d \sigma, & \tau>0, \\
v(\tau)=v_{0}+\frac{\mu}{\Gamma(\beta)} \int_{0}^{\tau} \frac{[u(\sigma)]^{p}}{(\tau-\sigma)^{1-\beta}} d \sigma, & \tau>0
\end{array}
$$

where $u_{0}>0, v_{0}>0, p>1, q>1,0<\alpha<\beta<1, \mu>0$. 
The form of (23) does not immediately allow for application of the results derived in Section 2. In order to apply the results of Section 2, it is necessary to first convert (23) into the form of (3). This is accomplished through the following change of variables:

$$
\begin{aligned}
\{u(\tau), v(\tau)\} & =\left\{u_{0}\left[u_{1}(t)+1\right], v_{0}\left[u_{2}(t)+1\right]\right\}, \\
\{\tau, \sigma\} & =\omega\{t, s\}, \\
\{\alpha, \beta\} & =\left\{\beta_{1}, \beta_{2}\right\}, \quad 0<\beta_{1}<\beta_{2}<1, \\
\left\{k_{1}(t), k_{2}(t)\right\} & =\gamma\left\{t^{\beta_{1}-1}, t^{\beta_{2}-1}\right\}, \\
\{q, p\} & =\left\{p_{1}, p_{2}\right\},
\end{aligned}
$$

where

$$
\gamma=\mu\left\{\frac{[\Gamma(\beta)]^{\alpha}}{[\Gamma(\alpha)]^{\beta}} \frac{\left[v_{0}\right]^{\beta q+\alpha}}{\left[u_{0}\right]^{\alpha p+\beta}}\right\}^{1 /(\beta-\alpha)}, \quad \omega=\left[\frac{\Gamma(\beta)}{\Gamma(\alpha)} \frac{v_{0}^{q+1}}{u_{0}^{p+1}}\right]^{1 /(\beta-\alpha)}
$$

This allows (23) to be expressed as

$$
u_{j}(t)=\int_{0}^{t} k_{j}(t-s)\left[u_{3-j}(s)+1\right]^{p_{j}} d s, \quad t>0, j=1,2 .
$$

The solution of (25) always experiences a blow-up as will be established in the following theorem.

Theorem 3.1. System (25) has a unique, continuous and monotonically increasing solution for $0 \leq t<t^{*}$. That solution ultimately experiences a blow-up as $t \rightarrow t_{c}<\infty$, where $t^{*} \leq t_{c} \leq t^{* *}$. Values of $t^{*}$ and $t^{* *}$ are given by

$$
t^{*}= \begin{cases}{\left[\frac{\beta_{1}}{\gamma} \frac{(r-1)^{(r-1)}}{r^{r}}\right]^{1 / \beta_{1}},} & \text { if } \frac{\beta_{1}}{\gamma} \frac{(r-1)^{(r-1)}}{r^{r}} \leq 1, \\ {\left[\frac{\beta_{2}}{\gamma} \frac{(r-1)^{(r-1)}}{r^{r}}-\frac{\left(\beta_{2}-\beta_{1}\right)}{\beta_{1}}\right]^{1 / \beta_{2}},} & \text { if } \frac{\beta_{1}}{\gamma} \frac{(r-1)^{(r-1)}}{r^{r}}>1,\end{cases}
$$

and

$$
t^{* *}= \begin{cases}{\left[\frac{\beta_{2}}{\gamma} \frac{r+1}{p_{1} p_{2}-1}\right]^{1 / \beta_{2}},} & \text { if } \frac{\beta_{2}}{\gamma} \frac{r+1}{p_{1} p_{2}-1} \leq 1, \\ {\left[\frac{\beta_{1}}{\gamma} \frac{r+1}{p_{1} p_{2}-1}+\frac{\left(\beta_{2}-\beta_{1}\right)}{\beta_{2}}\right]^{1 / \beta_{1}},} & \text { if } \frac{\beta_{2}}{\gamma} \frac{r+1}{p_{1} p_{2}-1}>1,\end{cases}
$$


where $r=\max \left\{p_{j}\right\}, j=1,2$.

Proof. The existence of a unique, continuous and monotonically increasing solution for $0 \leq t<t^{*}$ follows directly from Theorems 2.1 and 2.2. The value of $t^{*}$ follows by first noting that

$$
\bar{k}(t)=\max _{j=1,2} k_{j}(t)= \begin{cases}\gamma t^{-1+\beta_{1}}, & 0<t \leq 1 \\ \gamma t^{-1+\beta_{2}}, & 1<t<\infty\end{cases}
$$

and

$$
\bar{I}(t)=\int_{0}^{t} \bar{k}(s) d s=\frac{\gamma}{\beta_{1} \beta_{2}} \begin{cases}\beta_{2} t^{\beta_{1}}, & 0 \leq t \leq 1 \\ \beta_{1} t^{\beta_{2}}+\left(\beta_{2}-\beta_{1}\right), & 1<t<\infty\end{cases}
$$

Then note that $\bar{F}(M+1)=(M+1)^{r}, \bar{F}^{\prime}(M+1)=r(M+1)^{r-1}$. Then from (7) and (8),

$$
\bar{I}\left(t^{*}\right)=\frac{(r-1)^{r-1}}{r^{r}}<1
$$

Solving (30) for $t^{*}$ gives (26).

To calculate $t^{* *}$, first note that $\widetilde{k}(t)$ is the complementary case of (28), and hence

$$
\widetilde{I}(t)=\int_{0}^{t} \widetilde{k}(s) d s=\frac{\gamma}{\beta_{1} \beta_{2}} \begin{cases}\beta_{1} t^{\beta_{2}}, & 0 \leq t \leq 1 \\ \beta_{2} t^{\beta_{1}}-\left(\beta_{2}-\beta_{1}\right), & 1<t<\infty .\end{cases}
$$

For the calculation of $\kappa_{j}$, as defined by (16), it follows that (32)

$$
\kappa_{j}=\int_{1}^{\infty}\left[\frac{p_{3-j}+1}{\left(p_{j}+1\right)(y)^{p_{3-j}+1}+p_{3-j}-p_{j}}\right]^{p_{j} /\left(p_{j}+1\right)} d y, \quad j=1,2 .
$$

The integral $\kappa_{j}$ can be expressed in terms of hypergeometric functions. To obtain a more useful upper bound $t^{* *}$, it is convenient to replace (32) by

$$
\kappa_{j} \leq \int_{1}^{\infty}\left(\frac{1}{y}\right)^{\left(p_{1} p_{2}+r\right) /(1+r)} d y=\frac{r+1}{p_{1} p_{2}-1}, \quad j=1,2
$$


Thus $\kappa \leq(r+1) /\left(p_{1} p_{2}-1\right)$, from which (27) follows. It is clear that (27) is always satisfied by some $t^{* *}<\infty$. Hence, the solution of (25) always experiences a blow-up as $t \rightarrow t_{c}<\infty$.

Conversion of the bounds (26) and (27) back to the variables of (23) yields

$$
\begin{gathered}
\tau^{*}= \begin{cases}\omega\left[\frac{\alpha}{\gamma} \frac{(r-1)^{r-1}}{r^{r}}\right]^{1 / \alpha}, & \text { if } \frac{\alpha}{\gamma} \frac{(r-1)^{r-1}}{r^{r}} \leq 1, \\
\omega\left[\frac{\beta}{\gamma} \frac{(r-1)^{r-1}}{r^{r}}-\frac{(\beta-\alpha)}{\alpha}\right]^{1 / \beta}, & \text { if } \frac{\alpha}{\gamma} \frac{(r-1)^{r-1}}{r^{r}}>1,\end{cases} \\
\tau^{* *}= \begin{cases}\omega\left[\frac{\beta}{\gamma} \frac{r+1}{p q-1}\right]^{1 / \beta}, & \text { if } \frac{\beta}{\gamma} \frac{r+1}{p q-1} \leq 1, \\
\omega\left[\frac{\alpha}{\gamma} \frac{r+1}{p q-1}+\frac{(\beta-\alpha)}{\beta}\right]^{1 / \alpha}, & \text { if } \frac{\beta}{\gamma} \frac{r+1}{p q-1}>1 .\end{cases}
\end{gathered}
$$

It is difficult to make an exact comparison of the upper bound on the blow-up time determined in [3] with that provided in (35). The results of [3] involve a parameter used in establishing blow-up that has no counterpart in the proof of blow-up as presented in Theorem 2.3. In [3], an upper bound on the blow-up time is given by $\min \left[T_{u}, T_{v}\right]$, where $T_{u}$ depends upon $u_{0}$ and $T_{v}$ depends upon $v_{0}$. Converting those expressions to ones involving $\gamma$ and $\omega$ yields

$$
T_{u}=\hat{c_{u}} \omega \gamma^{-m}, \quad m=\frac{q+1}{\beta q+\alpha} ; \quad T_{v}=\hat{c_{v}} \omega \gamma^{-\tilde{m}}, \quad \widetilde{m}=\frac{p+1}{\alpha p+\beta},
$$

with $(1 / \beta) \leq m, \widetilde{m} \leq(1 / \alpha)$. The constants $\hat{c}_{u}$ and $\hat{c}_{v}$ are independent of $u_{0}$ and $v_{0}$. These expressions for $T_{u}$ and $T_{v}$ have some similarity to (35) in that they are directly proportional to $\omega$ and inversely proportional to a power of $\gamma$.

The growth profile of $u_{j}(t)$ near blow-up is derived by applying a methodology developed in $[\mathbf{1 0}]$. The results are provided in the following theorem.

Theorem 3.2. The asymptotic behavior near blow-up of the solution to (25) is given by

$$
u_{j}(t) \sim A_{j}\left(t_{c}-t\right)^{-l_{j}}, \quad \text { as } t \rightarrow t_{c},
$$


where

$$
l_{j}=\frac{p_{j} \beta_{3-j}+\beta_{j}}{p_{1} p_{2}-1}, \quad j=1,2,
$$

and

$$
A_{j}=\left[m_{j}\left(m_{3-j}\right)^{p_{j}}\right]^{1 /\left(p_{1} p_{2}-1\right)}, \quad m_{j}=\frac{\Gamma\left(p_{j} l_{3-j}\right)}{\gamma \Gamma\left(\beta_{j}\right) \Gamma\left(p_{j} l_{3-j}-\beta_{j}\right)} .
$$

Proof. This proof is a direct application of results developed in [10], which will be outlined below. Consider the change of variables

$$
w_{j}(\eta)=u_{j}(t), \quad \eta=\left(t_{c}-t\right)^{-1}-\eta_{0}, \quad \eta_{0}=t_{c}^{-1},
$$

whereby the blow-up $u_{j}(t) \rightarrow \infty$ as $t \rightarrow t_{c}$ then corresponds to $w_{j}(\eta) \rightarrow \infty$ as $\eta \rightarrow \infty$.

The change of variables converts $(25)$ to the form

$$
w_{j}(\eta)=\gamma \eta\left(\frac{\eta+\eta_{0}}{\eta}\right)^{1-\beta_{j}} \int_{0}^{1} \frac{\left[w_{3-j}(\eta \xi)+1\right]^{p_{j}}}{\left(\eta \xi+\eta_{0}\right)^{1+\beta_{j}}(1-\xi)^{1-\beta_{j}}} d \xi
$$

The methodology of $[\mathbf{1 0}]$ is based upon well-known asymptotic methods for Mellin transforms. To apply those methods, it is convenient to employ the Parseval formula for Mellin transforms so as to convert (40) to

$$
\begin{gathered}
w_{j}(\eta)=\gamma \Gamma\left(\beta_{j}\right) \eta\left(\frac{\eta+\eta_{0}}{\eta}\right)^{1-\beta_{j}} \frac{1}{2 \pi i} \\
\int_{c-i \infty}^{c+i \infty} \eta^{-z} \frac{\Gamma(1-z)}{\Gamma\left(1+\beta_{j}-z\right)} M\left[Q_{j}(\eta) ; z\right] d z,
\end{gathered}
$$

where the Mellin transform is defined by

$$
M\left[Q_{j}(\eta) ; z\right]=\int_{0}^{\infty} \eta^{z-1} Q_{j}(\eta) d \eta
$$

with

$$
Q_{j}(\eta)=\frac{\left[w_{3-j}(\eta)+1\right]^{p_{j}}}{\left(\eta+\eta_{0}\right)^{1+\beta_{j}}}
$$


The vertical integration path in (41) is taken to lie within a strip of analyticity of the integrand.

It is then shown in $[\mathbf{1 0}]$ that algebraic growth of $w_{j}(\eta)$ as given by

$$
w_{j}(\eta) \sim A_{j} \eta^{l_{j}}, \quad l_{j}>0, \quad A_{j}>0, \quad \text { as } \quad \eta \rightarrow \infty
$$

yields an asymptotic version of (41) in the form

$$
A_{j} \eta^{l_{j}} \sim \gamma \frac{\Gamma\left(\beta_{j}\right) \Gamma\left(p_{j} l_{3-j}-\beta_{j}\right)}{\Gamma\left(p_{j} l_{3-j}\right)}\left(A_{3-j}\right)^{p_{j}} \eta^{p_{j} l_{3-j}-\beta_{j}}, \quad j=1,2 .
$$

Satisfaction of (44) provides the values of $l_{j}$ and $A_{j}$ given by (37) and (38).

Remark. The asymptotic analysis of $[\mathbf{1 0}]$ does not determine an explicit value of $t_{c}$. The existence of $t_{c}$ with the bounds $t^{*} \leq t_{c} \leq$ $t^{* *}<\infty$ is provided by Theorem 3.1.

Converting the results of Theorem 3.2 back to the variables of (23) gives

$$
u(\tau) \sim c_{1}\left(\tau_{c}-\tau\right)^{-l_{1}}, \quad v(\tau) \sim c_{2}\left(\tau_{c}-\tau\right)^{-l_{2}}, \quad \text { as } \quad \tau \rightarrow \tau_{c},
$$

where

$$
\begin{aligned}
& c_{1}=\left[\frac{\Gamma\left(q l_{2}\right)}{\mu \Gamma\left(q l_{2}-\alpha\right)}\right]^{1 /(p q-1)}\left[\frac{\Gamma\left(p l_{1}\right)}{\mu \Gamma\left(p l_{1}-\beta\right)}\right]^{q /(p q-1)}, \\
& c_{2}=\left[\frac{\Gamma\left(q l_{2}\right)}{\mu \Gamma\left(q l_{2}-\alpha\right)}\right]^{p /(p q-1)}\left[\frac{\Gamma\left(p l_{1}\right)}{\mu \Gamma\left(p l_{1}-\beta\right)}\right]^{1 /(p q-1)} .
\end{aligned}
$$

These results for the blow-up profiles associated with the solution of (23) are essentially the same as those given in [3].

4. Application to a system with global and blow-up solutions. Consider the system

$$
u_{j}(t)=\int_{0}^{t} k_{j}(t-s)\left[u_{3-j}(s)+d_{j}\right]^{p_{j}} d s, \quad t>0, j=1,2,
$$


where $p_{1}=1, p_{2}=p>1, d_{1}=d>0$ and $d_{2}=1$. The kernel has the form

$$
k_{j}(t)=e^{-c t} t^{\beta_{j}-1}, \quad j=1,2,
$$

where $\beta_{1}=1, \beta_{2}=\alpha, 0<\alpha<1$ and $c \geq 0$.

Integral equations with kernels in the form of (48) have been considered in $[\mathbf{5}, \mathbf{6}]$. These papers demonstrate that, in a diffusive medium, the exponential factor arises from allowing for an advection effect. Typically, $c \sim v^{2}$, where $v$ is the velocity associated with advection. Advection has a cooling influence on the medium and would be expected to diminish the tendency for a blow-up to occur. It will be shown that, for $c$ sufficiently large, (47) has a global solution.

The special case of (47) in which $c=0$ can be converted to the integro-differential equation

$$
u^{\prime}(t)=\int_{0}^{t}(t-s)^{\alpha-1}[u(s)]^{p} d s+d, \quad t>0, u(0)=u_{0}>0 .
$$

This equation with $d=0$ has been considered in [4]. That limiting case will be discussed at the end of this section.

By applying the results of Section 2 to (47), a sufficient condition for a global solution is obtained. That condition involves the incomplete gamma function $\gamma(\alpha, x)=\int_{0}^{x} s^{\alpha-1} e^{-s} d s$. A global solution of (47) is provided by the following theorem.

Theorem 4.1. If $\alpha, d$ and $p$ are such that

$$
c^{-\alpha} \gamma(\alpha, c)+c^{-1} e^{-c}<p^{-p}\left\{\frac{p-1}{\max [1, d]}\right\}^{p-1},
$$

then (47) has a global solution.

Proof. To apply Theorem 2.1, it is noted that $\bar{k}(t)=e^{-c t} \max \left[1, t^{-1+\alpha}\right]$, and hence

$$
\bar{I}(t)= \begin{cases}c^{-\alpha} \gamma(\alpha, c t), & 0 \leq t \leq 1 \\ c^{-\alpha} \gamma(\alpha, c)+c^{-1}\left(e^{-c}-e^{-c t}\right), & t>1\end{cases}
$$


The value of $\Lambda$ follows from (7) with $\bar{F}(M+b)=(M+b)^{p}$, where $b=\max [1, d] \geq 1$. Then $M^{*}=b /(p-1)$, and hence

$$
\Lambda=p^{-p}\left\{\frac{p-1}{\max [1, d]}\right\}^{p-1}<1 .
$$

Then $\bar{I}(\infty)<\Lambda$ yields $(50)$.

It should be noted that (50) can always be satisfied for some sufficiently large value of $c$. From an application viewpoint, this implies that increasing the advection velocity can be used to prevent blow-up in a reactive-diffusive medium.

If $c$ is sufficiently small, (50) is not satisfied and hence allows for the possibility of a blow-up. If $c=0$, then the solution of (47) always experiences a blow-up as shown by the following theorem.

Theorem 4.2. Let $c=0$. Then the solution of (47) always experiences a blow-up as $t \rightarrow t_{c}$, where $t^{*} \leq t_{c} \leq t^{* *}<\infty$. The bounds on the blow-up time are given by

$$
t^{*}=\left[\alpha p^{-p}\left\{\frac{p-1}{\max [1, d]}\right\}^{p-1}\right]^{1 / \alpha}
$$

and

$$
t^{* *}= \begin{cases}\widetilde{\kappa}, & \text { if } \kappa<1, \\ {[\alpha(\widetilde{\kappa}-1)+1]^{1 / \alpha},} & \text { if } \kappa \geq 1,\end{cases}
$$

where

$$
\widetilde{\kappa}=\left[\frac{\pi}{2(p+1)}\right]^{1 / 2}\left[\Gamma\left(\frac{p-1}{2(p+1)}\right) / \Gamma\left(\frac{p}{p+1}\right)\right] .
$$

Proof. To apply Theorem 2.1, it is noted that $\bar{k}(t)=\max \left[1, t^{-1+\alpha}\right]$, and hence

$$
\bar{I}(t)= \begin{cases}t^{\alpha} / \alpha, & 0 \leq t \leq 1 \\ 1, & t>1\end{cases}
$$


Then $t^{*}$ is determined by $\bar{I}\left(t^{*}\right)=\Lambda$, where $\Lambda$ is given by (52). Since $\Lambda<1$, then $t^{*}=(\alpha \Lambda)^{1 / \alpha}<1$.

To determine $t^{* *}$, it is noted that $\widetilde{k}(t)=\min \left[1, t^{-1+\alpha}\right]$, and hence

$$
\widetilde{I}(t)= \begin{cases}t, & 0 \leq t \leq 1, \\ 1+\frac{t^{\alpha}-1}{\alpha}, & t>1 .\end{cases}
$$

For the lower bounding solution of (47), a version of (21) applicable to this problem is

$$
\frac{\left(V_{1}+1\right)^{p+1}}{p+1}-\frac{V_{2}^{2}}{2}=\frac{1}{p+1}-\frac{d^{2}}{2},
$$

where the initial values $V_{1}(0)=0, V_{2}(0)=d$ have been used. Using (58), expressions for $\kappa_{j}, j=1,2$ can be derived from (16). Calculations show that $\kappa_{1}<\kappa_{2}$, and hence

$$
\kappa=\left(\frac{p+1}{2}\right)^{1 / 2} \int_{0}^{\infty} \frac{d z}{\left[(z+1)^{p+1}-1+\frac{d^{2}(p+1)}{2}\right]^{1 / 2}} .
$$

An upper bound on $\kappa$ is given by $\widetilde{\kappa}$, which corresponds to (59) with $d=0$. Evaluation of (59) with $d=0$ provides (55). Then (54) follows from (55) and (57).

Remark. For the integro-differential equation (49) with $d=0$, as considered in [4], the results of Theorem 4.2 apply in the limit $d \rightarrow 0$. It follows from (53) that $t^{*}=\left[\alpha p^{-p}(p-1)^{p-1}\right]^{1 / \alpha}$. The upper bound $t^{* *}$ given by (54) is valid since $d=0$ was used to obtain $\kappa<\widetilde{\kappa}$.

For (47), the growth profile of $u_{j}(t)$ near blow-up is given by the following theorem.

Theorem 4.3. The asymptotic behavior near blow-up of the solution to (47) is given by Theorem 3.2 for the particular choice of parameters defined in (47), (48) and $\gamma=1$.

Proof. In the development of the methodology in [10], it was shown that the leading order growth of the solution near blow-up depends 
upon the asymptotic behavior of $k_{j}(t)$ as $t \rightarrow 0$. In this limit, the kernel in (48) is identical to that in (25) with $\gamma=1$. Hence the results of Theorem 3.2 apply to (47).

Remark. For the integro-differential equation (49) with $d=0$, it follows from Theorem 4.3 that

$$
u(t) \sim A\left(t_{c}-t\right)^{-l}, \quad \text { as } t \rightarrow t_{c},
$$

where

$$
l=\frac{1+\alpha}{p-1}, \quad A=\left[\frac{(1+\alpha) \Gamma\left[p\left(\frac{1+\alpha}{p-1}\right)\right]}{(p-1) \Gamma(\alpha) \Gamma\left(\frac{p+\alpha}{p-1}\right)}\right]^{1 /(p-1)}
$$

\section{REFERENCES}

1. H. Brunner and Z.W. Yang, Blow-up behavior of Hammerstein-type Volterra integral equations, J. Integral Equations Appl., to appear.

2. H. Hochstadt, Integral equations, Chapter 2, John Wiley \& Sons, New York, 1973.

3. M. Kirane and S.A. Malik, Profile of blowing-up solutions to a nonlinear system of fractional differential equations, Nonlinear Anal. 73 (2010), 3723-3736.

4. M. Kirane and S. Rihani, private communication, November 13, 2010.

5. C.M. Kirk and W.E. Olmstead, Blow-up in a reactive-diffusive medium with a moving heat source, Z. Angew. Math. Phys. 53 (2002), 147-159.

6. - Blow-up solutions of the two-dimensional heat equation due to a localized moving source, Anal. Appl. 3 (2005), 1-16.

7. T. Malolepzy and W. Okrasinski, Blowup time for solutions to some nonlinear Volterra integral equations, J. Math. Anal. Appl. 366 (2010), 372-384.

8. W.E. Olmstead, C.A. Roberts and K. Deng, Coupled Volterra equations with blow-up solutions, J. Integral Equations Appl. 7 (1995), 499-516.

9. C.A. Roberts, Recent results on blow-up and quenching for nonlinear Volterra equations, J. Comput. Appl. Math. 205 (2007), 736-743.

10. C.A. Roberts and W.E. Olmstead, Growth rates for blow-up solutions of nonlinear Volterra equations, Quart. Appl. Math. 54 (1996), 153-159.

California Polytechnic State University, San Luis Obispo, CA

Email address: ckirk@calpoly.edu

Northwestern University, Evanston, IL

Email address: weo@northwestern.edu

Department of Mathematics and Computer Science, College of the Holy Cross, Worcester, MA 01610

Email address: croberts@holycross.edu 